\title{
Promoting change in medical practice
}

I t's often held that innovations come to the Canadian health care system at an absolute snail's pace. Certainly the nation's health ministers were of that opinion when they asked the Health Council of Canada to create some sort of mechanism to disseminate information about new developments and practices.

But the end result - a website called the Health Innovation Portal (www. healthcouncilcanada.ca/innovation) has one medical information technology expert, Dr. Karim Keshavjee, pondering whether there's much value in simply curating articles about innovative practices, when they haven't been fully run through an evidentiary gamut.

Nevertheless, the sharing of innovative practices across the country may help bring all jurisdictions up to speed in a notoriously fragmented health care system, says Mark Dobrow, director of analysis and reporting for the council. "Everyone can potentially benefit. I think there's a lot of people in bigger provinces like Ontario that are not aware of what's happening from one region to the other, because there's not always a systematic way to share that type of information. And so this is one of the ways that we can do it."

The portal fills a gap in the dissemination of potentially valuable knowledge, particularly "grey literature," that doesn't fit into the traditional scholarly publishing process, he says. Submissions are evaluated for entry based on an "innovative practices evaluation framework," rather than through randomized control trials, meta-analyses or systematic reviews of evidence.

"The types of practices that we're profiling in this don't have that type of evidence base to support them, generally. So if we tried to apply that type of model or rigorous approach to it, nothing would make it through the framework," Dobrow adds. "Essentially, anybody [can] submit something, but they do have to provide information to support it, and that is the basis for the framework."

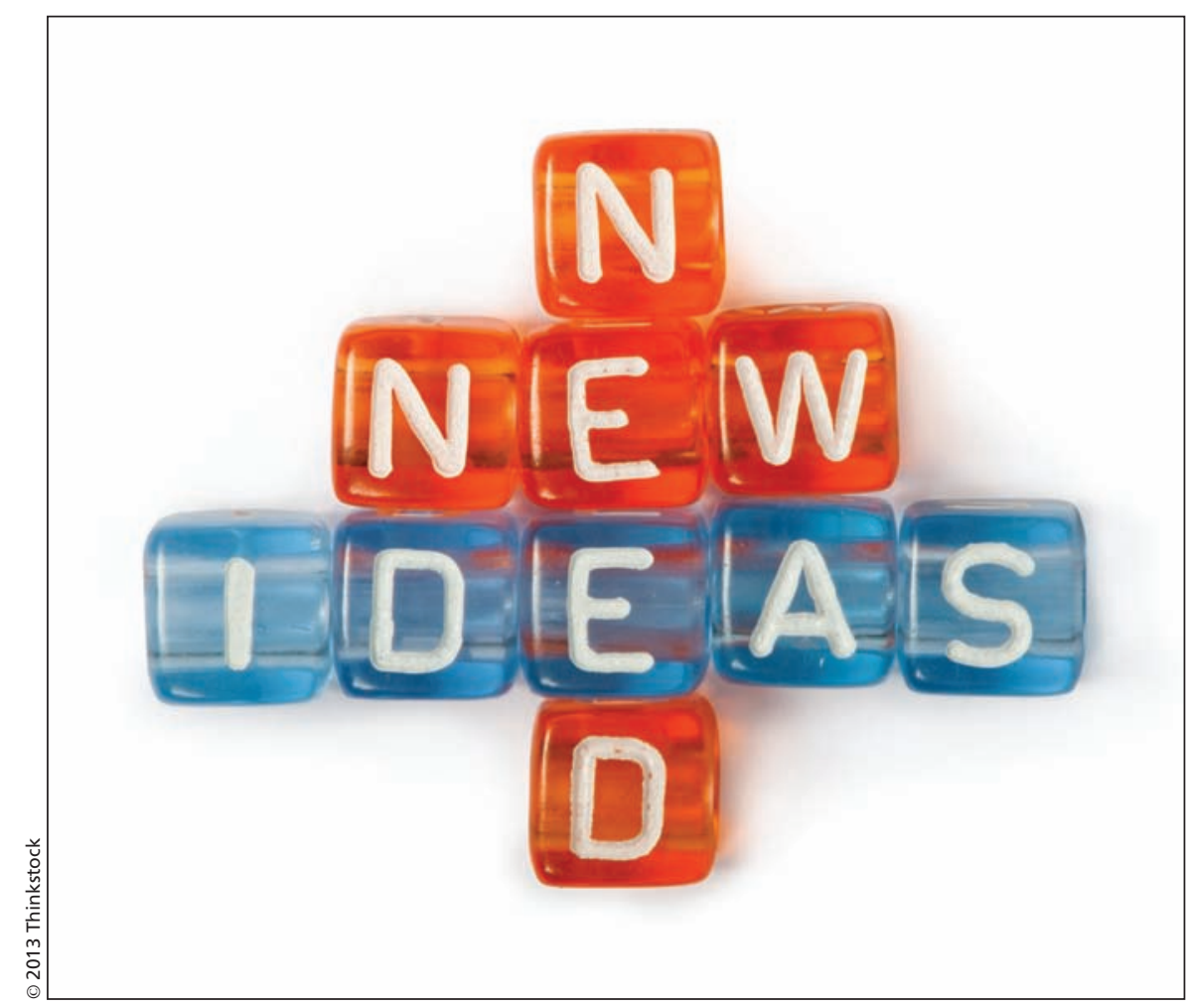

It's hoped that sharing innovative practices among Canadian jurisdictions will reduce fragmentation in the health care system.

To be eligible for evaluation, a practice must meet inclusion criteria, to wit: "Relevance — the practice addresses a need or gap related to a current health care issue, including those identified by a federal, provincial, or territorial government; Non-Medical — the practice is not a specific drug, surgical, or medical intervention that warrants an evaluation through other processes, such as a randomized controlled trial, systematic review, and/or meta-analysis; and Resonance - the practice is perceived as new and innovative by those for whom the practice is intended, those delivering the practice, and/or other key stakeholders in the health care system" (http ://innovation.healthcouncilcanada.ca/sites /default/files/Page\%20PDFs/IP_Frame work_Eng_final.pdf). If a practice meets inclusion criteria, it is then evaluated according to following criteria: "Quality of Evidence - the extent to which a practice has been evaluated by high quality evidence; Impact — the extent to which a practice has results that demonstrate a positive impact on health outcomes and/or health care system performance; Applicability — the extent to which a practice has been implemented or adapted for use outside of its original setting; and Transferability - the extent to which the results of a practice have been replicated outside of its original setting."

That's all well and good, but don't expect over-worked physicians to get too excited about a database that likely contains information that may be of limited utility to their practices, says Dr. Karim Keshavjee, a family physician and associate member of the Centre for Evaluation of Medicines, an independent academic research institute in Hamilton, Ontario. "I think it's a waste of time. It's nice but not very useful because people who are busy have to come and read it. There's no 
accepted metrics. There's no engagement of the community."

Physicians don't make decisions to change their practices in a vacuum, adds Keshavjee, who is also CEO of Toronto, Ontario-based InfoClin, a firm that helps physicians adopt information technology systems. The process of change requires debate and connection with other practitioners within a social context, which the database can't provide, he says. "There has to be a more robust plan of action than just curating articles - and curating articles that are of questionable validation and evidence. They have to contextualize it within the experiences of the various stakeholders who are interested in making this work and saying, 'Here's how we can make this work for all of us'."
The database contained 267 entries on practices, policies, programs and services from domestic and international health care experts as of Jan. 22. Almost all are reports, though there are several podcasts. Eventually, the plan is to add videos and web seminars. Most entries, 235, are not categorized. Those remaining are labelled as "emerging," "promising" or "leading."

Among examples of innovations that have already been included in the database are a British initiative that embeds self-management support within mainstream health services (identified as an "emerging" practice); a description of how First Nations communities in Manitoba benefit from telehealth (a "promising" practice); and Stanford University's Chronic Disease Self-Management Program (a "leading" practice.
But will an innovation adopted in Saskatchewan, whether emerging, promising or leading, necessarily benefit physicians in Quebec or Ontario?

No, suggests Keshavjee. There are other factors to consider, such as differing levels of funding on areas that provinces have deemed to be priorities.

"How are people going to implement these things when the resources required to do them are in some senses insurmountable?" Keshavjee asks. "I don't see that this is really addressing any of the issues that they care about. I think part of the issue that the health council has to deal with is that they have to present this material in a way that connects it to the individual doctors." - Adam Miller, CMAJ

CMAJ 2013. DOI:10.1503/cmaj.109-4370 